\title{
Calculation of Low-Frequency Vibrational Modes of Biologically Important Isomers
}

\author{
Inke Jones, Tamath J. Rainsford, Bernd M. Fischer, and Derek Abbott \\ Centre of Biomedical Engineering and Department of Electrical \& Electronic Engineering, \\ The University of Adelaide, SA 5005, Australia
}

\begin{abstract}
In this paper we compare the value of different molecular modeling techniques for the prediction of vibrational modes, especially in the mid- and far-infrared region. There is a wide range of different levels of theory available for molecular modelling - the choice depending on the kind of system to be investigated. For our calculations we use different theoretical approaches such as Hartree-Fock and Density functional theory. We also compare the performances of two available electronic structure programs - Gamess-US and Gaussian03. As examples, we use two different retinoids - all-trans retinal and all-trans retinoic acid - derivatives of Vitamin A.
\end{abstract}

Keywords: Vibrational spectroscopy, ab initio molecular modelling, density functional theory, computational chemistry, electronic structure programs, biomolecules, retinoids

\section{INTRODUCTION}

Being able to distinguish between different isomers of a particular molecule is very important in various fields of science such as biology, medicine and also pharmaceutical industry. For example, retinoids, a class of chemical compounds that are related to vitamin A, play an important role in vision, bone growth, in the treatment of skin lesions and acne, and as chemotherapy for certain types of leukemia. Many different geometric isomers of retinol, retinal and retinoic acid are possible as a result of either a trans or cis configuration of the four $\mathrm{C}=\mathrm{C}$ double bonds found in the polyene chain. The far- and mid-infrared spectral regions are very important for the investigation of biologically important molecules, since rotational and vibrational transitions of such molecules lie in these ranges. An a priori knowledge of the expected spectra enables the comparison of theoretical and experimental data and allows for improved experimentation through the interpretation of data.

Molecular modelling techniques have long been versatile tools in vibrational spectroscopy of biomolecules. ${ }^{1,2}$ In a previous study ${ }^{3}$ we investigated the use of ab initio molecular orbital calculations of frequencies in the far-infrared region for the identification of biomolecules. However, the assignment of distinct vibrational modes using those calculations remains difficult, mainly because the agreement between theoretical and experimental data, especially in the far-Infrared region, is often not very consistent. There is a wide range of molecular modelling and quantum chemistry techniques available. Ab initio molecular orbital theory, which does not depend on experimental input data, uses Schrödinger's equation to directly and quantitatively predict chemical phenomena requiring as input only the masses and charges of electrons and nuclei. Commonly, molecular orbitals, expressed as linear combinations of a finite set of one-electron basis functions, are used to approximate a full wavefunction. Density functional theory (DFT) on the other hand uses the electron density as its functional and therefore takes electron correlation into account. Typical electronic structure programs for such calculations are Gamess-US ${ }^{4}$ and Gaussian03. ${ }^{5}$ In this paper we investigate the influence of different theoretical approaches on the reliability of calculated vibrational frequencies.

Further author information: (Send correspondence to I. Jones)

E-mail: ijones@eleceng.adelaide.edu.au,dabbott@eleceng.adelaide.edu.au

Biomedical Applications of Micro- and Nanoengineering III, edited by Dan V. Nicolau, Proc. of SPIE Vol. 6416, 641607, (2006) - 1605-7422/06/\$15 - doi: 10.1117/12.695404 


\section{COMPUTATIONAL CHEMISTRY- $A B$ INITIO MOLECULAR ORBITAL THEORY}

Computational chemistry uses mathematical descriptions of chemistry incorporated into efficient computer programs to calculate the structures and properties of molecules. The basis of electronic structure methods is the assumption that all chemistry can be described in terms of the interactions between electronic charges within molecules. The quantum mechanical description of chemical bonds is given by a space- and timedependent probability distribution: the molecular wavefunction. The molecular wavefunction is defined by the Schrödinger equation. ${ }^{6}$ In principle, it is possible to solve the Schrödinger equation, in either its timedependent form or time-independent, but in practice this is only possible for very small systems. Therefore, a great number of approximate methods try to achieve the best trade-off between accuracy and computational cost. Ab initio techniques are the most accurate, do not depend on experimental data and are broadly useful for studying systems composed of tens of atoms; however, they are computationally expensive. Semiempirical methods are not as accurate, as they employ extensive approximations and require experimental input parameters, but are not so computationally demanding and are useful for moderate sized systems. Very large systems, containing thousands of atoms, can be studied with molecular mechanics methods based on classical physics. They are computationally fast but require experimental inputs and have limited accuracy. Recently hybrid methods combining high-level quantum mechanical calculations on a small part of the system with lower-level methods on the remainder have been used. ${ }^{7}$

In general, the choice of an appropriate quantum mechanical model is always a compromise. The most sophisticated approach available is certainly expected to yield the most accurate results. On the other hand, the usage of a particular model will most likely be restricted by the size of the system. Quantum mechanical models can broadly be classified in two dimensions, one referring to the level of treatment of electron correlation and the other to the size of the basis set expansion.

\subsection{Ab Initio Methods}

In our previous paper ${ }^{3}$ we have described the basic principle of ab initio molecular orbital theory based on the approximate solution of Schrödinger's equation. To completely describe the quantum mechanical behaviour of electrons in a system it is necessary to calculate the many-electron wavefunction for this particular system. This may be obtained from the time-independent Schrödinger equation in principle, but in practice each electron is influenced by the behaviour of all the other electrons in the system. ${ }^{8}$ To solve the Schrödinger equation directly for all these electrons it would be required to solve a great number of differential equations simultaneously, depending on the size of the system. Molecular orbital theory is an approach to molecular quantum mechanics which uses one-electron functions or orbitals to approximate a full wavefunction. A molecular orbital is a function of the cartesian coordinates $x, y$ and $z$ of a single electron, its square being the probability distribution of the electron in space. The complete wavefunction for a single electron is described by the product of a molecular orbital and a spin function.

Hartree-Fock (HF) analysis is the simplest type of ab initio electronic structure calculation, which describes each electron in a molecule as moving in the average electric field generated by the other electrons and nuclei. $^{9} \quad$ As a single-particle theory, HF theory systematically overestimates molecular energies because it neglects the correlated motion of electrons resulting from Coulomb interactions. Although there is no exact analytical solution to the time-independent molecular Schrödinger equation for systems containing more than one electron, approximate solutions can be obtained using standard numerical techniques. The approach of all $a b$ initio techniques is to build the total wavefunction from a "basis" set of mathematical functions capable of reproducing critical properties of the system. ${ }^{6}$ In the simplest of HF models, the number of basis functions needed to describe each atom will be as small as possible, but large enough to accommodate all electrons and still maintain spherical symmetry. This also means that the molecular orbitals will have only limited flexibility. Hence, the larger the basis set the higher the number of adjustable coefficients in the variational procedure, which leads to a more improved description of the molecular orbital. The Hartree-Fock limit represents the best that can be achieved with a single-electron configuration. 


\subsection{Density Functional Methods}

As mentioned above, traditional methods in electronic structure theory such as Hartree-Fock (HF) theory are based on the complicated many-electron wavefunction and neglect the correlation between the electrons of the system. In density functional theory (DFT), the electron correlation is modelled as a functional ${ }^{10}$ of the electron density. Here, the ground-state of the system is considered to be defined by that electron density distribution, which minimizes the total energy of the system. While the many-body wavefunction is dependent on $3 N$ variables, three spatial variables for each of the $N$ electrons, the density is only a function of three variables, which are needed to define the charge density at a particular point. Also, the ground-state electron density uniquely determines all other ground state properties of the system, particularly the ground-state energy. ${ }^{8}$ The most common implementation of density functional theory is through the Kohn-Sham method. This method partitions the electronic energy via the Kohn-Sham equations ${ }^{8,11}$ into kinetic energy, potential energy, and the electron-electron repulsion term; all of which represent the classical energy of the electron distribution. The last partition of these equations is the electron correlation term. This term represents both the quantum mechanical exchange energy, which accounts for electron spin, ${ }^{12}$ and the dynamic correlation energy due to the concerted motion of individual electrons. Pure DFT methods calculate the electron correlation term by pairing an exchange functional with a correlation functional and are therefore designated by the choice of combination. ${ }^{6}$ There are three general categories of DFT: local density approximations (LDA), generalized gradient approximations (GGA), and "hybrid" combinations of DFT and Hartree-Fock terms. An example of the latter is B3LYP, which combines Hartree-Fock self-interaction corrections with density functional exchange and correlation. B3 denotes Becke's three-parameter hybrid functional, ${ }^{13}$ while "LYP" is a gradient-corrected correlation functional developed by Lee, Yang and Parr. ${ }^{14}$ Density functional methods have the advantage of avoiding the computational expense of other post-HF methods by approximating the effects of electron correlation. Given its demonstrated accuracy for predicting molecular energies and activation barriers, the enormous amount of time saved by using DFT makes it an attractive method for studying the ground state properties and reactions of larger energetic molecules. ${ }^{6}$

\subsection{Basis Sets}

In general, a basis set is a range of mathematical functions used to solve a differential equation. In quantum chemical calculations, the term "basis set" is used to describe a collection of a finite set of one-electron functions (Gaussians) representing atomic orbitals, which are optimized to reproduce the desired chemical properties of a system. ${ }^{6}$ Initially, the atomic orbitals used were Slater-type orbitals (STOs), which correspond to a set of functions that decay exponentially with distance from the nuclei. Later, it was realized that these Slater-type orbitals could in turn be approximated as linear combinations of Gaussian orbitals instead. Today, there are hundreds of basis sets composed of Gaussian-type orbitals (GTOs). The choice of basis set determines the level of accuracy and depends on two components: (a) the size of the basis set and (b) the treatment of electron correlation. In general the choice of basis set will always be a compromise. The simplest level of ab initio molecular orbital theory involves the use of minimal basis sets, which are typically composed of the minimum number of basis functions required to represent all of the electrons on each atom. The largest basis sets can contain literally dozens to hundreds of basis functions on each atom. The number of basis set functions per atom is dependent on the kinds of atoms involved in the calculation.

An example of a minimal basis set is the STO-XG basis set. It consists of expansions of Slater-type atomic orbitals in terms of X gaussian functions. In these basis sets, the same number of Gaussian primitives comprise core and valence orbitals. Typically, minimal basis sets such as STO-3G and STO-6G give rough results that are insufficient for research-quality publication, but are much cheaper than their larger counterparts. But since it is usually the valence electrons which principally take part in molecular bonding, it is common to represent valence orbitals by more than one basis function. This can be realized by using split-valence basis sets such as the Pople split valence sets $6-21 \mathrm{G}$ and $6-31 \mathrm{G}$. In the case of $6-31 \mathrm{G}$, the "6" represents the number primitive Gaussians comprising each core atomic orbital basis function. The " 3 " and "1" indicate that the valence orbitals are composed of two basis functions each, where the first one is composed of a linear combination of three primitive Gaussian functions and the second of one primitive Gaussian function. ${ }^{9}$ 
There are other important additions to the above mentioned basis sets available such as polarization basis functions and diffuse basis functions. Polarization basis functions take the influence of the nuclei in an molecule on each other into account, which will distort or polarize the electron density near the nuclei. Orbitals that have more flexible shapes in a molecule than the s, p, d, etc. shapes in the free atoms are needed. This is accomplished by adding a p-shaped orbital to a s-shaped orbital (or equivalently a d-orbital to a p-orbital), which will "shift" the resulting orbital sideways, polarize it and thus allow for small displacements of the center of electronic charge away from the nuclear positions. The polarization function 6-31G* (or 6-31G(d)) adds a set of $\mathrm{d}$ functions to the atoms in the first and second rows $(\mathrm{Li}-\mathrm{Cl})$ and $6-31 \mathrm{G}^{* *}($ or $6-31 \mathrm{G}(\mathrm{d}, \mathrm{p})$ ) adds a set of $\mathrm{d}$ functions to the atoms in the first and second rows $(\mathrm{Li}-\mathrm{Cl})$ and a set of $\mathrm{p}$ functions to hydrogen. Diffuse basis functions are a useful addition in cases of excited states and in anions where the electronic density is more spread out over the molecule.

\subsection{Sources of Error}

As discussed above, Hartree-Fock theory is a single-particle approximation and therefore cannot adequately treat the correlated motion of electrons that occurs due to Coulombic electron-electron interactions. Neglect of electron correlation is believed to result in systematic HF errors, such as underestimated bond lengths and overestimated vibrational frequencies. ${ }^{6}$ Many types of calculations begin with a HF calculation and subsequently correct for the electronic correlation. Examples of these methods are configuration-interaction (CI) methods, Møller-Plesset (MP) perturbation theory. But each of these techniques should be considered carefully since most of them violate at least one of the fundamental criteria that define the $a b$ initio methodology. For example, truncated CI calculations (i.e., anything less than full configuration interaction) are not size-consistent, so comparative CI studies should include added corrections. ${ }^{15}$ At the same time, MP perturbation theory is not a variational technique, and as such does not provide an upper bound to the true molecular energy. The problem in density functional theory is that the exact functional dependence of the electron correlation term upon the electron density is unknown and must be approximated by assuming that it has certain properties (e.g., it behaves like a uniform electron gas). Excited-state DFT calculations, which require the application of linear response theory, have so far been limited to atomic systems. Another source of error is that molecular vibration calculations in electronic structure programs do not automatically take anharmonicity into account. This typically leads to an over estimation of the frequencies. However, this overestimation seems to be relatively uniform and therefore generic frequency scaling factors can be applied. ${ }^{16}$ For far-IR calculations, this does not seem to be applicable. Not only do the calculated modes appear to be shifted, there are also modes additional to the ones experimentally detected. This could be due to molecular rotations and translations that are located in the millimeter wave region. Further studies are needed to investigate these findings.

In general, $a b$ initio molecular orbital studies on complex systems should begin with calculations using small basis sets. This obtains a timely qualitative assessment of molecular properties, and determines the computational cost of the higher-order calculations. However, minimal basis sets like STO-3G should only be used when the size of the molecule is beyond the scope of available computer resources for even small split-valence sets like 3-21G. ${ }^{6}$ Since larger basis sets contain more adjustable parameters and are thus better able to approximate true molecular wavefunctions, quantitative accuracy improves with the size of basis sets. Polarization functions improve the accuracy of fundamental basis sets (e.g., changing 6-31G to 6-31G(d)), although the ultimate gain is limited. ${ }^{17}$ Diffuse functions (e.g., changing 6-31G to 6-31G+) will improve results under certain circumstances.

\subsection{Electronic Structure Programs}

To perform an ab initio molecular orbital calculations, a graphic interface is need in which the particular molecule can be build. There is a wide range of electronic structure programs available, some of which are free of charge (e.g. Molden ${ }^{18}$ ) and some require a purchasable licence (e.g. GaussView ${ }^{19}$ ). Through building a particular molecule in one of these programs, a matrix containing all information about bond lengths and bond angles within that molecule is produced. This matrix can then be used as the input for the desired calculations performed by electronic structure programs. Typical engines used for this are Gamess ${ }^{4,20}$ (Generalized Atomic 
and Molecular Electronic Structure System) and Gaussian.5,19 Gamess is available in two different versions, Gamess-UK, ${ }^{20}$ which is only freely available to U.K. academics, and Gamess-US, ${ }^{4}$ which is available on the internet as freeware to both academic and industrial users all over the world. A licence for Gaussian, here Gaussian $03,{ }^{5}$ is available to academic and commercial users. Both programs can predict the energies, molecular structures, and vibrational frequencies of molecular systems, along with numerous molecular properties derived from these basic computation types and they are both available as unix or windows versions. For details we would like to refer to the individual manuals. To perform calculations in Gamess-US it is necessary for the user to write a Fortran based input code, including all desired specifications. If one acquires Gaussian03 in combination with GaussView, ${ }^{19}$ the two programs are linked with each other, using a "button-click" interface to allow the user to select all specifications for the calculation after building the molecule. The program then creates its own input code based on these parameters.

\section{THE RETINOIDS}

Retinoids are chemical derivatives of the dietary supplement vitamin A, formed through oxidation reactions. Retinal or retinaldehyde is fundamental in the transduction of light into visual signals in the photoreceptor level of the retina. For further information we refer to our previous work ${ }^{3}$ where we have described the function of the retinal and its isomers in great detail. Retinoic acid is a carotenoid organic compound that is a component of visual pigments. It causes cell differentiation, and plays a part in the growth and development of embryos. Medicinally, it is used in tretinoin creams for the treatment of acne and other skin disorders. It is also a component of many commercial products that are advertised as being able to slow skin aging or remove wrinkles. ${ }^{21}$ In addition, it is also used to treat at least one form of leukemia by causing the blood cells to differentiate, mature and die. ${ }^{22}$ Similar to retinal, retinoic acid occurs in different isomeric form, which all have different properties and applications. In paper we focus on the all-trans isomers of both retinal and retinoic acid (see Figure 1).

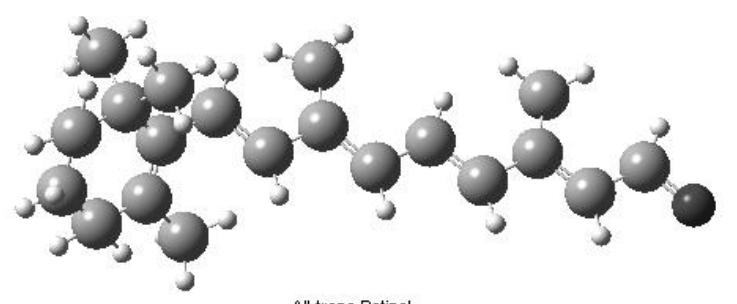

All-trans Retinal

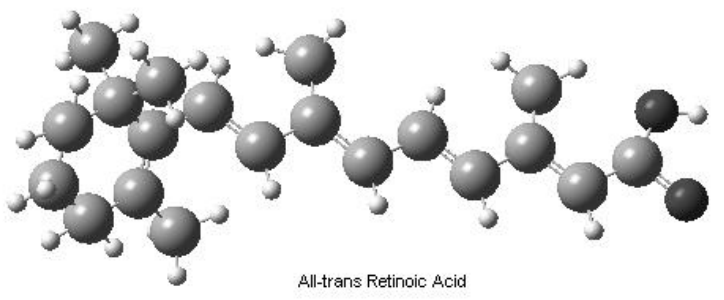

Figure 1. All-trans retinal and all-trans retinoic acid isomers visualized in GaussView ${ }^{19}$

\section{RESULTS AND DISCUSSION}

We used the two electronic structure programs Gamess-US and Gaussian03 to optimize the geometries of 3 different molecules and to calculate their vibrational frequencies. All three molecules where built in GaussView 
and the same input matrices where used for the calculations in these electronic structure programs. With both programs we performed calculations using both unrestricted HF theory and DFT. Using Gamess-US we found the best approach to be to start with a small basis set (STO-3G) and a higher gradient convergence tolerance and then further optimize the geometry using a smaller convergence tolerance and higher level basis sets (6-31G, $6-31 \mathrm{G}^{*}$ and $6-31 \mathrm{G}^{* *}$ ) to achieve geometry convergence and to locate a reliable energy minimum. The frequency calculations of the optimized structure where then performed using the basis set of the last optimization (6-31G in the case of DFT and 6-31G** in the case of HF). For Gaussian03 we found, in agreement with previous studies, it not necessary to perform many optimization steps based on the experiences of previous studies, ${ }^{1,23}$ but only carried out one combined optimization and frequency calculation for each molecule and theory (6-31G in the case of DFT and 6-31G** in the case of HF). The experimental data of the all-trans retinal and all-trans retinoic acid molecules in the far-infrared region $\left(10-110 \mathrm{~cm}^{-1}\right)$ was obtained using an Infrared Spectrometer of the type Bruker IFS120HR fitted with a LHe cooled silcon Bolometer detector, where the samples were run as pressed dry powder on polyethylene (PE) plates. For the mid-infrared region $\left(400-1900 \mathrm{~cm}^{-1}\right)$ we used a Perkin Elmer BX-1 Fourier-Transform Infrared Spectrometer with a 1mW helium-neon unit and to measure the vibrational frequencies of both of these molecules pressed into $\mathrm{KBr}$ discs.

Our results show that the experimental data in the mid-infrared region agrees very well with the calculated data overall (see Figures 2(a) and 3(a)). From Figure 2(a) it can be seen that the calculated data in the mid-IR region using the density functional approach (B3LYP), even with a smaller basis set (6-31G), agrees even better with the experimental data than the results of our Hartree-Fock calculation with a larger basis set (6-31G**). Using the same basis set together with B3LYP the calculations would be computationally much more expensive. It should be noted that we achieved a great improvement in the results of our HF calculations using the basis set $6-31 \mathrm{G}^{* *}$ as compared to $6-31 \mathrm{G}^{*}$ and $6-31 \mathrm{G}$, which is therefore not demonstrated here. The results of the two electronic structure programs (see Figure 2(b)) show a very good agreement between the experimental data and the B3LYP calculations of both Gamess-US and Gaussian03. When comparing the graphs in detail we found that there seems to be slightly better agreement between the IR data and the results acquired from Gamess-US. This could be explained by the extended optimization steps involved in the calculations, but since it is such a minor improvement, the computational time Gaussian03 saves might outweigh this in comparison. Figure 3(a) and (b) confirm these findings. As can be seen from Figure 4(a) and (b), our far-IR calculations and our experimental data do not agree as well as it was observed in the mid-infrared region. Graph (a) shows a slight agreement between the experimental data and the Hartree-Fock approach, but this is not confirmed by graph (b). These findings could be due to the fact that spectral features in the far-IR region are usually due to intermolecular vibrations as opposed to intramolecular vibrations in the mid-infrared region. In our calculations we only considered one isolated molecule, hence there are no intermolecular vibrations possible. 


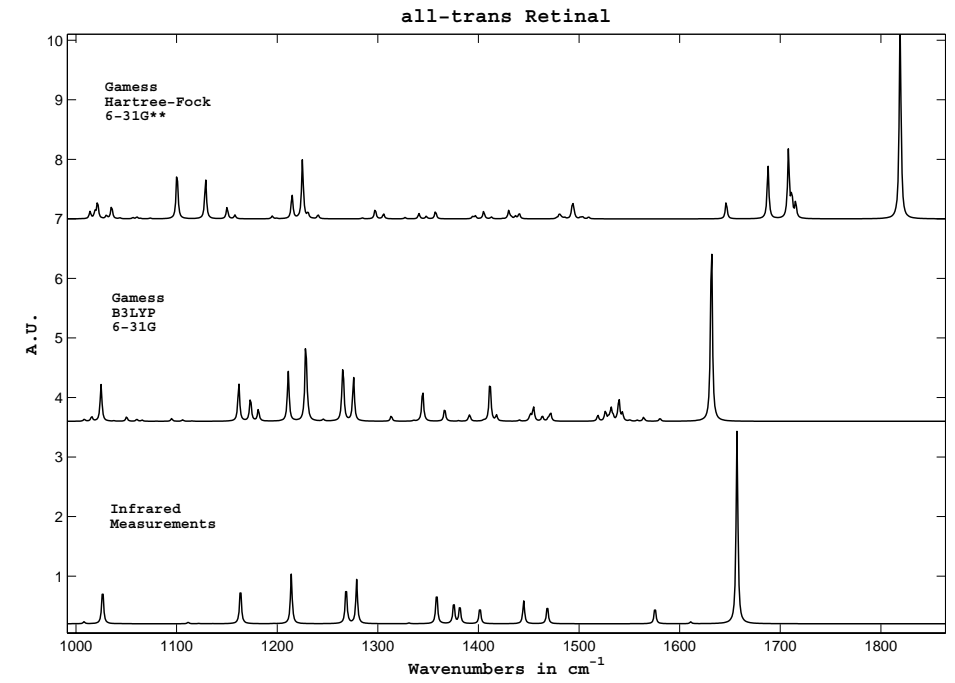

(a)

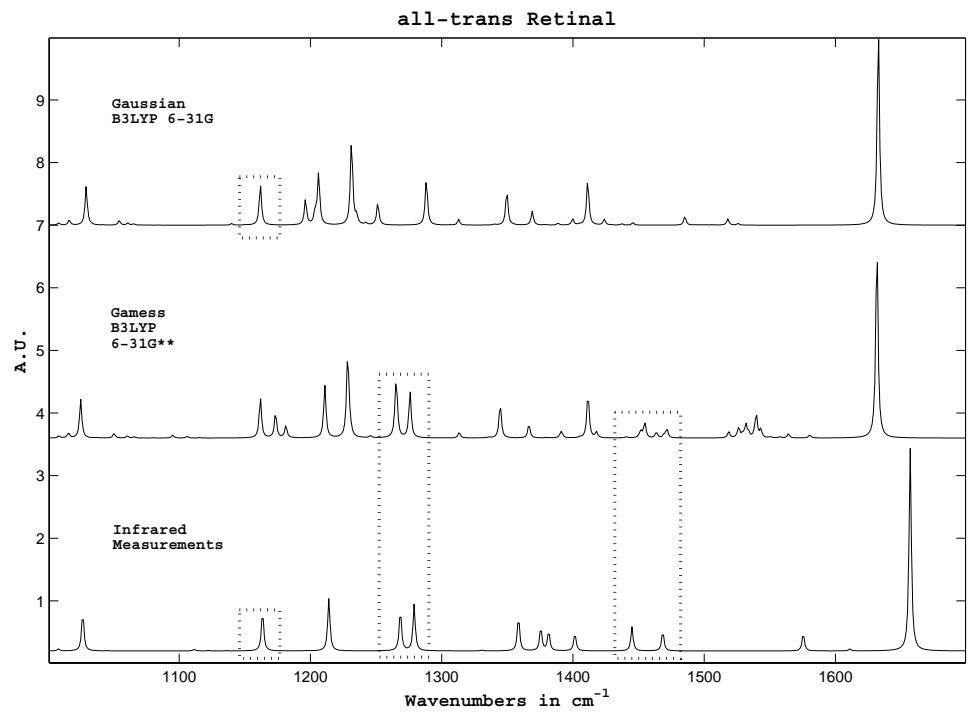

(b)

Figure 2. Calculated vibrational spectra of all-trans retinal in comparison to our own experimental infrared data covering the mid-IR region of approximately $1000-1900 \mathrm{~cm}^{-1}$. It should be noted that we already achieved a great and successive improvement of the HF calculations by changing the basis set from $6-31 \mathrm{G}$ to $6-31 \mathrm{G}^{*}$ and finally to 6-31G**, thus we do not include these results here.The intensities are shown in arbitrary units, with the intensities of the experimental data fitted to the calculated results and the individual traces were shifted vertically for better presentation. (a) Shows the results of different levels of theory(Hartree-Fock and B3LYP) using the electronic structure package Gamess-US ${ }^{4}$ and (b) shows the results of B3LYP calculations using both electronic structure packages (Gamess$\mathrm{US}^{4}$ and Gaussian $03^{5}$ ). 


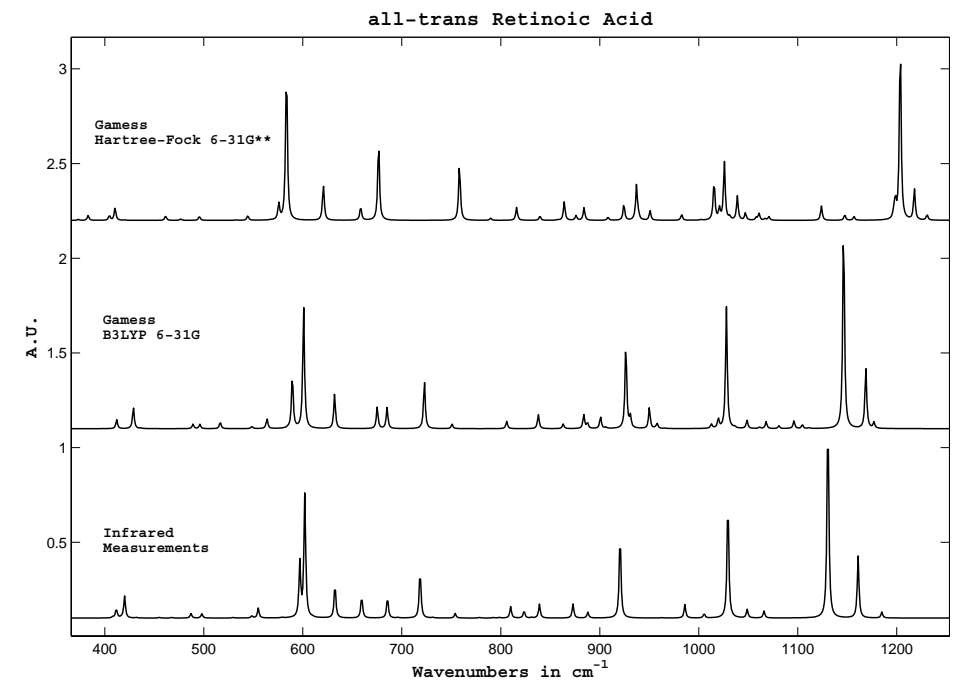

(a)

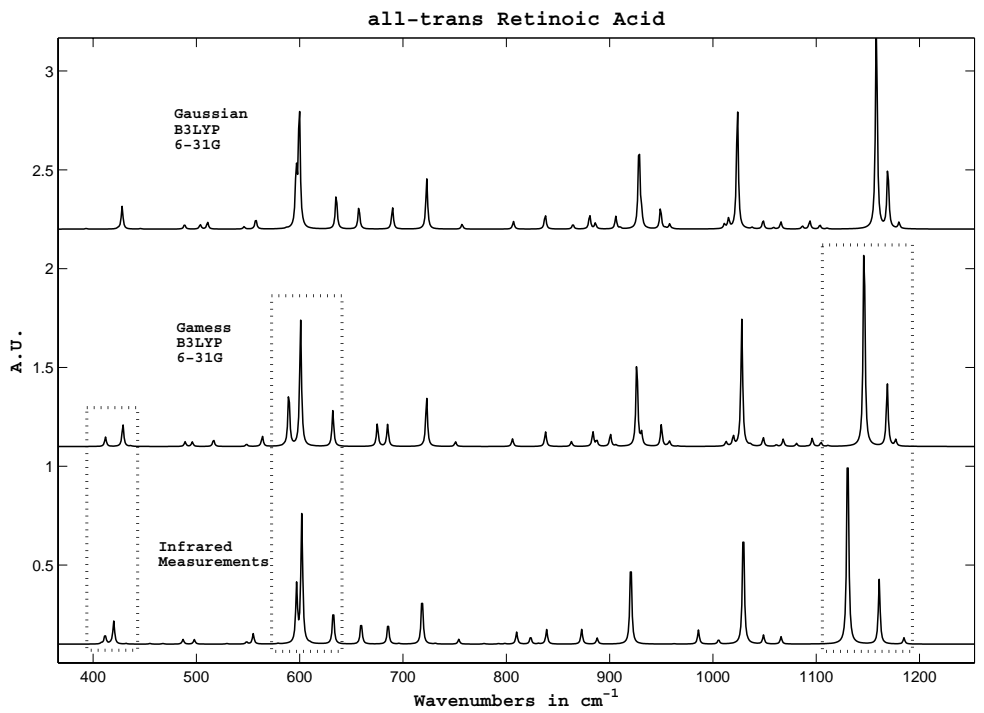

(b)

Figure 3. Calculated vibrational spectra of all-trans retinoic acid in comparison to our own experimental infrared data covering the mid-IR region of approximately $400-1200 \mathrm{~cm}^{-1}$. It should be noted that we already achieved a great and successive improvement of the HF calculations by changing the basis set from 6-31G to 6-31G* and finally to 6-31G**, thus we do not include these results here.The intensities are shown in arbitrary units, with the intensities of the experimental data fitted to the calculated results and the individual traces were shifted vertically for better presentation. (a) Shows the results of different levels of theory(Hartree-Fock and B3LYP) using the electronic structure package Gamess-US ${ }^{4}$ and (b) shows the results of B3LYP calculations using both electronic structure packages (Gamess$\mathrm{US}^{4}$ and Gaussian $03^{5}$ ). 


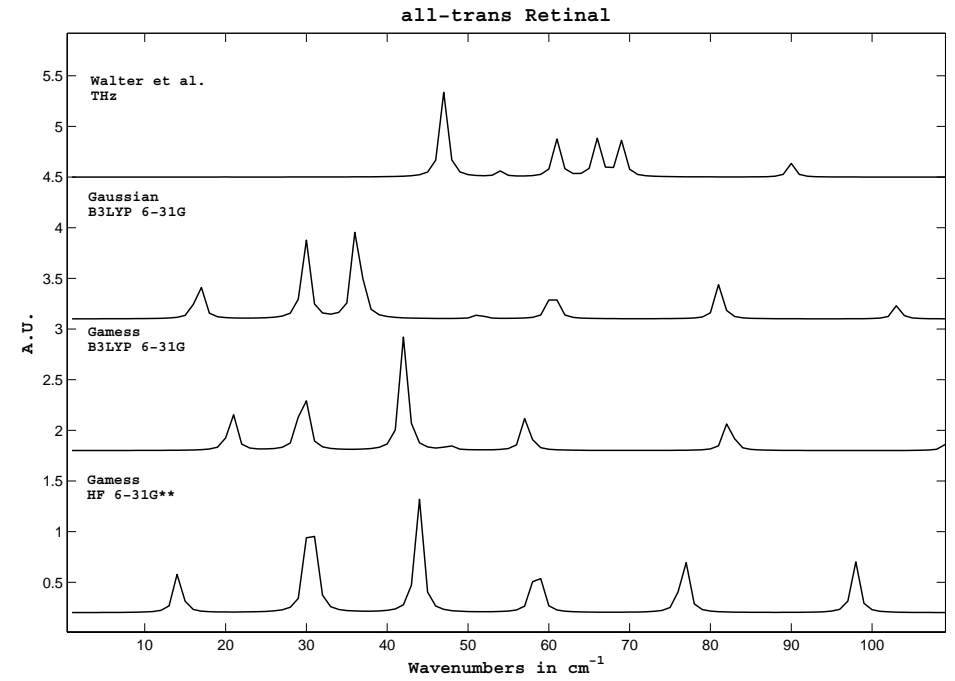

(a)

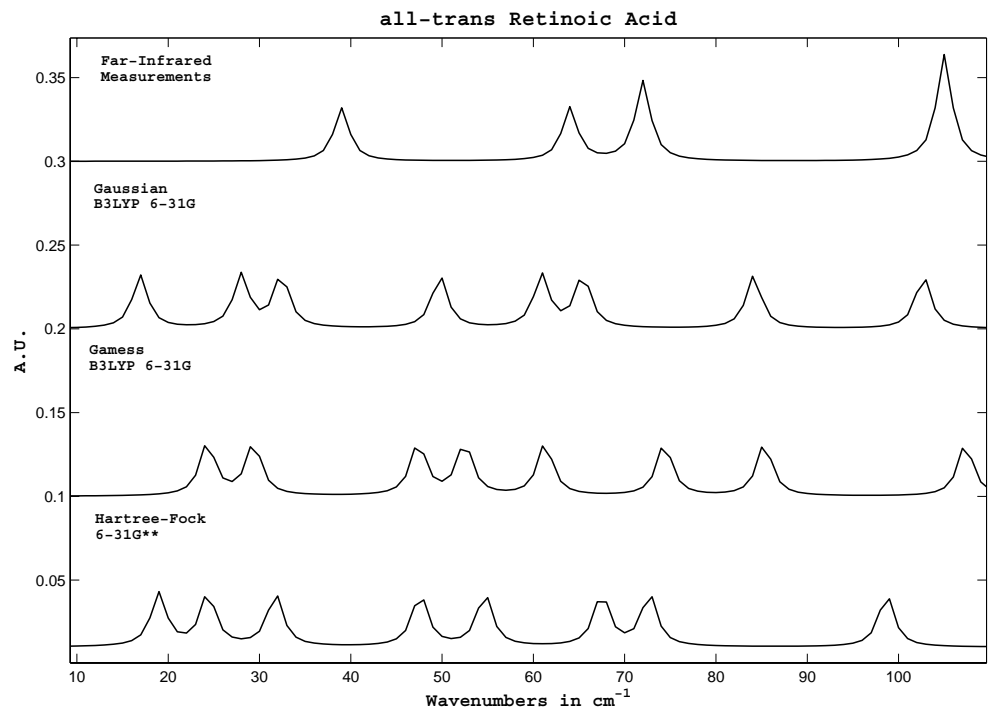

(b)

Figure 4. Calculated low-frequency vibrational spectra using the electronic structure packages Gamess-US and Gaussian03 and different levels of theory (Hartree-Fock and B3LYP), covering the far-IR region of approximately 10-110 $\mathrm{cm}^{-1}$. It should be noted that we already achieved a great and successive improvement of the HF calculations by changing the basis set from $6-31 \mathrm{G}$ to $6-31 \mathrm{G}^{*}$ and finally to $6-31 \mathrm{G}^{* *}$, thus we do not include these results here.The intensities are shown in arbitrary units, with the intensities of the experimental data fitted to the calculated results and the individual traces were shifted vertically for better presentation. (a) Shows the calculated spectra of all-trans retinal in comparison to experimental infrared data reported in Walther et al ${ }^{23}$ and (b) Shows the calculated spectra of all-trans retinoic acid in comparison to experimental infrared data reported here. 


\section{CONCLUSION}

We have performed ab initio molecular orbital calculations using the Hartree-Fock approach and density functional theory, applying a range of different basis sets. We then compared the results to experimental infrared data. Additionally, we investigated the advantages and disadvantages of the two electronic structure programs Gamess-US and Gaussian03. We found that the theoretical vibrational modes in the mid-infrared region agree well with the experimental data. The density functional B3LYP approach $(6-31 \mathrm{G})$ was shown to produced more accurate results than Hartree-Fock theory, even when using a higher level basis set (6$\left.31 \mathrm{G}^{* *}\right)$. As for the electronic structure programs, it seemed that in our particular examples Gamess-US ${ }^{4}$ provided slightly more accurate results, whereas Gaussian $03^{5}$ was found to be more user friendly, especially in combination with the visualization program GaussView, ${ }^{19}$ and delivered considerably faster results. Further studies on a wide range of molecules are needed to confirm these findings. The calculated vibrational modes in the far-IR region however did not seem to coincide very well with the experimental data overall. This could be due to a variety of reasons, such as anharmonic vibrations, intermolecular vibrations and temperature dependency, all of which were not taken into account by the theoretical approaches and need to be investigated through further studies. Future work could also include the investigation of the influence of the choice of electronic structure program on the optimized molecule and its minimum energy, i.e. do the many steps in the optimization process in Gamess lead to a different optimized molecule than the combined optimization and frequency calculation method in Gaussian, and which of these structures is more realistic?

\section{ACKNOWLEDGEMENT}

The authors would like to thank Finlay Shanks, School of Chemistry, Faculty of Science, Monash University, Melbourne, for reporting the far-infrared measurements and Gregory Metha, School of Chemistry \& Physics, the University of Adelaide, for his guidance and support with our infrared measurements. Funding from the Australian Research Council (ARC) and the Sir Ross and Sir Keith Smith Fund is gratefully acknowledged.

\section{REFERENCES}

1. F. L. Gervasio, G. Cardini, P. R. Salvi, and V. Schettino, "Low-frequency vibrations of all-trans-retinal: Far-infrared and raman spectra and density functional calculations," Journal of Physical Chemistry A 102, pp. 2131-2136, 1998.

2. A. Bifone, H. J. M. deGroot, and F. Buda, "Ab initio molecular dynamics of retinals," Chemical Physics Letters 248, pp. 165-172, 1996.

3. I. Jones, T. J. Rainsford, B. Fischer, and D. Abbott, "Towards T-ray spectroscopy of retinal isomers: A review of methods and modelling," Vibrational Spectroscopy Sp. Iss. SI 41(2), pp. 144-154, 2006.

4. M. W. Schmidt, K. K. Baldridge, J. A. Boatz, S. T. Elbert, M. S. Gordon, J. H. Jensen, S. Koseki, N. Matsunaga, K. A. Nguyen, S. Su, T. L. Windus, M. Dupuis, and J. A. Montgomery, "General atomic and molecular electronic structure system," Journal of Computational Chemistry 14, pp. 1347-1363, 1993.

5. M. J. Frisch et al., Gaussian 03, Revision B 0.5, Gaussian Inc., Wallingford CT, 2004.

6. H. Dorsett and A. White, "Overview of molecular modelling and ab initio molecular orbital methods suitable for use with energetic materials," tech. rep., DSTO Aeronautical and Maritime Research Laboratory, 2000.

7. R. D. J. Froese and K. Morokuma, "Accurate calculations of bond breaking energies in c60 using the three layered oniom method," Chemical Physics Letters 305, pp. 419-424, 1999.

8. R. G. Parr and W. Yang, Density-Functional Theory of Atoms and Molecules, vol. 16 of International Series of Monographs on Chemistry, Oxford University Press, New York, 1989.

9. W. J. Hehre, L. Radom, P. v. R. Schleyer, and J. A. Pople, Ab Initio Molecular Orbital Theory, John Wiley and Sons, Inc., New York, 1986.

10. Y. Akutsu and S. Y. Tahara, "Calculations of heats of formation for nitro compounds by semi-empirical mo methods and molecular mechanics," Journal of Energetic Materials 9, pp. 161-172, 1991. 
11. D. Joubert, Density Functionals: Theory and Applications, Springer-Verlag, Heidelberg, 1998.

12. H. Bergman, H. Östmark, K. Ekvall, and A. Langlet, "A study of the sensitivity and decomposition of keto-RDX," Proceedings of the 10th Symposium (International) on Detonation, pp. 862-869, 1993.

13. A. D. Becke, "Density functional exchange energy approximation with asymptotic behaviour," Physical Review A38(6), pp. 3098-3100, 1998.

14. C. Lee, W. Yang, and R. G. Parr, "Development of the Colle-Salvetti correlation energy formula into a functional of the electron density," Physical Review B37(2), pp. 785-789, 1988.

15. W. J. Hehre, L. Radom, P. v. R. Schleyer, and J. A. Pople, Handbook of Computational Quantum Chemistry, Oxford University Press, New York, 1998.

16. A. P. Scott and L. Radom, "Harmonic vibrational frequencies: An evaluation of Hartree-Fock, MøllerPlesset, quadratic configuration interaction, density functional theory, and semiempirical scale factors," Journal of Physical Chemistry 100, pp. 16502-16513, 1996.

17. E. P. Wallis and D. L. Thompson, "Molecular dynamics simulations of ring inversion in RDX," Journal of Chemical Physics 99(4), pp. 2661-2673, 1993.

18. G. Schaftenaar and J. H. Noordik, "Molden: a pre- and post-processing program for molecular and electronic structures," Journal of Computer-Aided Molecular Design 14, pp. 123-134, 2000.

19. J. A. Wass, "Modeling molecules," Science 290(5499), pp. 2098-2098, 2000.

20. M. F. Guest, I. J. Bush, H. J. J. van Dam, P. Sherwood, J. M. H. Thomas, J. H. van Lenthe, R. W. A. Havenith, and J. Kendrick, "The GAMESS-UK electronic structure package: algorithms, developments and applications," Molecular Physics 103(6-8), pp. 719-747, 2005.

21. O. Sorg, C. Antille, G. Kaya, and J. H. Saurat, "Retinoids in cosmeceuticals," Dermatologic Therapy 19, p. $289296,2006$.

22. N. Mirza, M. Fishman, I. Fricke, M. Dunn, A. M. Neuger, T. J. Frost, R. M. Lush, S. Antonia, and D. I. Gabrilovich, "All-trans-retinoic acid improves differentiation of myeloid cells and immune response in cancer patients," Cancer research 66, pp. 9299-9307, 2006.

23. M. Walther, B. Fischer, M. Schall, H. Helm, and P. U. Jepsen, "Far-infared vibrational spectra of alltrans retinal, 9-cis and 13-cis retinal measured by THz time-domain spectroscopy," Chemical Physics Letters 332, pp. 389-395, 2000. 